\title{
Population Pharmacokinetic Analysis and Model-Based Simulations of Aripiprazole for a 1-Day Initiation Regimen for the Long-Acting Antipsychotic Aripiprazole Lauroxil
}

\author{
Marjie L. Hard ${ }^{1}$ Angela Y. Wehr ${ }^{1} \cdot$ Brian M. Sadler $^{2} \cdot$ Richard J. Mills $^{3}$. \\ Lisa von Moltke ${ }^{1}$
}

Published online: 11 June 2018

(C) The Author(s) 2018

\begin{abstract}
Background and Objectives Aripiprazole lauroxil (AL), a long-acting injectable antipsychotic for the treatment of schizophrenia, requires 21 days of oral aripiprazole supplementation upon initiation (21-day initiation regimen). An alternative 1-day initiation regimen utilizing a nano-crystalline milled dispersion of $\mathrm{AL}\left(\mathrm{AL}_{\mathrm{NCD}}\right)$ plus a single $30 \mathrm{mg}$ oral aripiprazole dose achieved aripiprazole concentrations associated with therapeutic doses of aripiprazole in the same time frame as the 21-day initiation regimen when starting AL (441 or $882 \mathrm{mg}$ ). A population pharmacokinetic (PopPK) model was developed to describe aripiprazole pharmacokinetics following administration of $\mathrm{AL}_{\mathrm{NCD}}, \mathrm{AL}$ and oral aripiprazole, and evaluate dosing scenarios likely to be encountered in clinical practice.

Methods In total, 12,768 plasma aripiprazole concentrations from 343 patients (from 4 clinical studies) were
\end{abstract}

Electronic supplementary material The online version of this article (https://doi.org/10.1007/s13318-018-0488-4) contains supplementary material, which is available to authorized users.

Lisa von Moltke

Lisa.vonMoltke@alkermes.com

1 Alkermes, Inc., 852 Winter Street, Waltham, MA 02451, USA

2 ICON, Gaithersburg, MD, USA

3 ICON, Marlow, UK included in the PopPK analysis and used to construct the model.

Results Concomitant administration of the 1-day initiation regimen with all approved AL dosing regimens (441, 662, or $882 \mathrm{mg}$ monthly, $882 \mathrm{mg}$ every 6 weeks, or $1064 \mathrm{mg}$ every 2 months) is predicted to achieve aripiprazole concentrations associated with therapeutic doses of $\mathrm{AL}$ using the 21-day initiation regimen within 4 days, maintaining these concentrations until the next AL dose. Administration of the first $\mathrm{AL}$ injection 10 days after the 1-day initiation regimen resulted in median aripiprazole concentrations just before the second dose of $\mathrm{AL} \geq 77 \%$ of that when coadministered on the same day. Coadministration of $\mathrm{AL}$ with a single $\mathrm{AL}_{\mathrm{NCD}}$ injection was predicted to be effective in rapidly re-establishing concentrations associated with therapeutic doses of AL following dose delay.

Conclusions Model-based simulations demonstrate that the 1-day initiation regimen is suitable for starting treatment with all $\mathrm{AL}$ doses, allowing a window of $\leq 10$ days between initiation and $\mathrm{AL}$ administration. $\mathrm{AL}_{\mathrm{NCD}}$ may also be used to re-establish concentrations associated with therapeutic doses of AL in conjunction with a delayed AL dose. 


\section{Key Points}

$\mathrm{AL}_{\mathrm{NCD}}$ is a nano-crystalline milled dispersion formulation of aripiprazole lauroxil (AL) developed to be administered in conjunction with a single $30 \mathrm{mg}$ oral aripiprazole tablet in a single day (the 1-day initiation regimen), as a potential alternative to the 21-day oral aripiprazole daily supplementation currently required to initiate patients onto AL.

Model-based simulations show that the 1-day initiation regimen can be used with any of the five approved AL dosing regimens, such that it will be possible to provide up to 2 months of therapeutic treatment without any oral supplementation beyond Day 1.

AL can be administered on the same day or up to 10 days after the 1-day initiation regimen, thus allowing for flexible dosing. $\mathrm{AL}_{\mathrm{NCD}}$ is a suitable alternative to 7 days oral aripiprazole supplementation to re-establish aripiprazole concentrations within the range associated with therapeutic doses of AL following a missed AL dose.

\section{Introduction}

Preventing or delaying relapse is a major goal in the treatment of schizophrenia [1]. Research on the epidemiology of relapse in schizophrenia showed a public health crisis of $>350,000$ schizophrenia admissions in general hospital settings in 2012 [2]. In addition, $>1$ in 5 patients discharged with schizophrenia are readmitted within 30 days of discharge [2]. Relapse is associated with many complications; it can jeopardize the safety of the patient and the community and can impair the patient's social functioning and other important recovery goals. Antipsychotic medications are recommended for all phases of schizophrenia and play an essential role in lowering the likelihood of relapse. Their potential benefits, however, are diminished by medication gaps. Even brief medication gaps as short as a few days will decrease the effectiveness of antipsychotics for the prevention of relapse [1]. Long-acting injectable (LAI) formulations of antipsychotics are available and offer some advantages compared with oral medications, including decreased risk for unintentional or deliberate overdose, and they allow the monitoring of patients' adherence status, which is helpful in the prevention of medication gaps [3]. Despite these advantages, treatment initiation with LAIs can be complicated by delayed release of the drug, resulting in a delay in achieving therapeutic or steady state concentrations immediately after administration [3]. In these cases, oral doses are required to supplement the level of circulating drug to therapeutic levels and might have to be taken for 2-3 weeks. A shorter initiation time might be preferable for patients with schizophrenia, who are particularly vulnerable to nonadherence and relapse when transitioning from inpatient to outpatient care.

Aripiprazole lauroxil (AL), a prodrug of aripiprazole, is an LAI antipsychotic medication indicated for the treatment of schizophrenia [4-6]. AL can be administered at a dose of 441, 662, or $882 \mathrm{mg}$ monthly, an $882 \mathrm{mg}$ dose every 6 weeks, or a $1064 \mathrm{mg}$ dose every 2 months [4]. Given the delayed release of aripiprazole after the first injection of AL, a concurrent 21-day course of oral aripiprazole (21-day initiation regimen) is required to rapidly achieve plasma concentrations within the range associated with therapeutic doses of aripiprazole (as observed in previous studies) [7,8].

To provide an alternative option to daily oral supplementation during this critical initiation period, a nano-crystalline milled dispersion of $\mathrm{AL}$ (known as $\mathrm{AL}_{\mathrm{NCD}}$ ) was developed. $\mathrm{AL}_{\mathrm{NCD}}$ is composed of nanometersized particles and has faster dissolution properties than standard AL (composed of micron-sized particles), resulting in earlier appearance of circulating plasma aripiprazole and leading to more rapid achievement of therapeutic levels. Based on results of an earlier pharmacokinetic study [9], coadministration of a single dose of $\mathrm{AL}_{\mathrm{NCD}}$ and a single $30 \mathrm{mg}$ dose of oral aripiprazole in 1 day (1-day initiation regimen) was as effective as the 21-day initiation regimen when initiating AL (441 or $882 \mathrm{mg}$ ), whereby aripiprazole concentrations within the range associated with therapeutic doses of AL using the 21-day initiation regimen were achieved within 4 days of treatment initiation, and were maintained for the full 28-day period until the next AL dose. In that study, only 2 of the 4 currently approved AL doses (441, 662, 882, and $1064 \mathrm{mg}$ ) were evaluated with the 1-day initiation regimen. Here we report the development of a population pharmacokinetic (PopPK) model using pharmacokinetic data from 4 Phase 1 studies to describe the pharmacokinetics of aripiprazole after intramuscular (IM) injection of $\mathrm{AL}_{\mathrm{NCD}}$ and $\mathrm{AL}$ and administration of oral aripiprazole. This model was used to perform PK simulations to evaluate the use of the 1-day initiation regimen with the other approved AL regimens and to address questions related to the use of $\mathrm{AL}_{\mathrm{NCD}}$ in treatment scenarios likely to be encountered in clinical practice. 


\section{Methods}

\subsection{Population Pharmacokinetic Model}

\subsubsection{Data Set}

The PopPK model for aripiprazole was developed using data from 4 Phase 1 studies, 3 of which examined and demonstrated the feasibility of using $\mathrm{AL}_{\mathrm{NCD}}$ as an initiation regimen for AL [9]. These studies (ALK9072-B101, ALK9072-B102, ALK9072-B103) are referred to here as Study 1, Study 2, and Study 3, respectively; specifically, Studies 1 and 3 evaluated $\mathrm{AL}_{\mathrm{NCD}}$ dosing alone, and Study 2 evaluated dosing of $\mathrm{AL}_{\mathrm{NCD}}, \mathrm{AL}$, and $30 \mathrm{mg}$ oral aripiprazole. The fourth study was an earlier Phase 1 study in which AL was administered alone (ALK9072-A105, referred to as Study 4). All studies were carried out in accordance with the Declaration of Helsinki; a brief description of the studies is provided in Supplementary Table S1 (Online Resource 1). Each study enrolled adult patients with schizophrenia or schizoaffective disorder whose conditions were stable with a first-line antipsychotic medication (excluding aripiprazole). Patients were excluded from Studies 1, 2 and 3 if they were cytochrome P450 (CYP) 2 D6 poor metabolizers (PM). Patients who received potent CYP3A4 inducers or inhibitors or CYP2D6 inhibitors (prescription medications, over-the-counter medications, or dietary supplements), within the 30 days prior to admission were also excluded from the studies. All patients with sufficient data on dosing, actual sampling time, and aripiprazole concentration were included in the PopPK analysis.

Patient baseline characteristics were comparable across the different studies (Table 1). The mean ( \pm standard deviation) patient age was $45.2 \pm 10.8$ years; $73 \%$ were men, $78 \%$ were Black or African American, and mean body weight was $89.1 \pm 17.9 \mathrm{~kg}$ (Table 1). In total, 12,768 plasma aripiprazole concentrations (including 351 [3\%] records that were below the lower limit of quantitation) from 343 patients were included in the analysis, and the M3 method [10] was employed. The data set contained 2536 dosing records (1742 oral aripiprazole, 626 AL, 168 $\left.\mathrm{AL}_{\mathrm{NCD}}\right)$.

\subsubsection{AL ${ }_{N C D}$ PopPK Model Development}

The model was developed using nonlinear mixed-effects modeling with NONMEM version 7.3.0 (ICON Gaithersburg, MD, USA); PDx-Pop version 5.2 was used as the NONMEM interface (full details of the development of the PopPK model are provided in the Supplementary Text, Online Resource 2). For modeling, $\mathrm{AL}$ and $\mathrm{AL}_{\mathrm{NCD}}$ doses were expressed as aripiprazole equivalents of 75, 150, 300, 450, 600, and $724 \mathrm{mg}$ (corresponding to $\mathrm{AL}_{\mathrm{NCD}}$ or $\mathrm{AL}$ doses of 110, 221, 441, 662, 882, and $1064 \mathrm{mg}$, respectively). The model was built in a sequential manner as the data became available in stages (as the individual studies were completed). A previously developed PopPK model for AL [11] that included an oral input function served as the starting point for model development and was expanded to include an input function for $\mathrm{AL}_{\mathrm{NCD}}$. $\mathrm{A}$ base model that appropriately described the time course of aripiprazole plasma concentrations in Study 1, accounting for IM absorption of $\mathrm{AL}_{\mathrm{NCD}}$ and conversion to aripiprazole, was initially selected. The initial base model was expanded after the inclusion of final data from Study 3 (completed before Study 2) and was used to re-estimate model parameters. On receipt of the final data from Study 2, the model was updated, existing parameters were re-estimated, and full covariate analysis was performed.
Table 1 Patient baseline characteristics for the final PopPK analysis

\begin{tabular}{|c|c|c|c|c|c|}
\hline \multirow[t]{2}{*}{ Covariate } & \multicolumn{5}{|l|}{ Study } \\
\hline & $\begin{array}{l}\text { All studies } \\
N=343\end{array}$ & $\begin{array}{l}\text { Study } 1 \\
n=41\end{array}$ & $\begin{array}{l}\text { Study } 2 \\
n=161\end{array}$ & $\begin{array}{l}\text { Study } 3 \\
n=47\end{array}$ & $\begin{array}{l}\text { Study } 4 \\
n=94\end{array}$ \\
\hline Mean age, years, (SD) & $45.2(10.8)$ & $44.2(11.5)$ & $44.0(10.6)$ & $48.6(9.9)$ & $46.1(11.1)$ \\
\hline Male, $n(\%)$ & $251(73)$ & $32(78)$ & $118(73)$ & $34(72)$ & $67(71)$ \\
\hline \multicolumn{6}{|l|}{ Ethnicity, $n(\%)$} \\
\hline Hispanic or Latino & $18(5)$ & $5(12)$ & $6(4)$ & $2(4)$ & $5(5)$ \\
\hline Other & $325(95)$ & $36(88)$ & $155(96)$ & $45(96)$ & $89(95)$ \\
\hline \multicolumn{6}{|l|}{ Race, $n(\%)$} \\
\hline Black or African American & $267(78)$ & $33(80)$ & $125(78)$ & $37(79)$ & $72(77)$ \\
\hline White & $73(21)$ & $8(20)$ & $35(22)$ & $9(19)$ & $21(22)$ \\
\hline Other & $3(1)$ & 0 & $1(1)$ & $1(2)$ & $1(1)$ \\
\hline Mean weight, kg (SD) & $89.1(17.9)$ & $89.0(19.4)$ & $89.4(18.9)$ & $86.6(15.6)$ & $89.8(16.8)$ \\
\hline
\end{tabular}

PopPK population pharmacokinetics, $S D$ standard deviation 
Covariates available for evaluation included the continuous covariates of age (years) and body weight $(\mathrm{kg})$ at baseline, and categorical covariates of injection site (gluteal or deltoid), formulation, CYP2D6 genotype, ethnicity, sex, and race. Body weight, formulation, and CYP2D6 genotype were evaluated as part of the initial base model development with Study 1 . The covariate model was re-evaluated after receipt of Study 2 and Study 3 data. Effects of body weight on clearance (CL) and volume (V) terms were incorporated using fixed allometric exponents of 0.75 and 1 , respectively, and scaled to $70 \mathrm{~kg}$. The full model with backwards deletion approach was utilized for covariate modeling. All covariate-parameter relationships of interest were entered in the model simultaneously. Highly correlated covariates were tested in separate models to avoid confounding the estimation of covariate effects. A backwards deletion was carried out at the $p=0.001$ [increased objective function value (OFV) less than 10.83 points, degrees of freedom = 1] significance level where the relative influence of each covariate on the model was re-evaluated by deleting it from the full model on an individual basis.

Data from Study 4 were then added (to include data from administration of AL alone), and parameters of the final model were updated based on the data from all 4 studies. The final model was evaluated using goodness-of-fit analyses and by generating prediction-corrected visual predictive checks (pcVPCs). As the M3 method was employed, individual weighted residuals (IWRES) were used rather than conditional weighted residuals (CWRES). The predictive performance of the final models was assessed by applying a posterior pcVPC and calculating the percentage of the observations outside the $90 \%$ prediction intervals (PIs).

\subsubsection{Model Refinement for Simulations}

Initial simulations for oral aripiprazole dosed at $15 \mathrm{mg}$ for 21 days suggested that the final PopPK model may overpredict the exposure to aripiprazole with multiple oral doses. Investigations showed that the variability in aripiprazole concentrations associated with the 21-day initiation regimen in Study 2 was high, but consistent with what was previously observed in the pivotal Phase 3 study [6] and that there appeared to be a subgroup of patients whose profiles reflected lower exposure after multiple administrations of oral aripiprazole. A mixture model was evaluated for oral aripiprazole to determine whether a subpopulation could be formally identified (see Supplementary Text, Online Resource 2).

\subsection{PopPK Simulations}

The final PopPK model and mixture model for oral aripiprazole were used as appropriate to perform Monte Carlo simulations using Pharsight Trial Simulator version 2.2.2 (Certara USA, Inc., Princeton, NJ, USA). In total, 500 individual concentration-time profiles were simulated for each scenario without incorporation of the residual error from the final PopPK model. Simulations were conducted to evaluate the impact on aripiprazole concentrations when the 1-day initiation regimen was coadministered with $\mathrm{AL}$ on the same day, in contrast to administering AL several days later. Simulations were conducted for all 5 approved AL dosing regimens [441, 662, $882 \mathrm{mg}$ every 4 weeks (q4wk); $882 \mathrm{mg}$ q6wk; $1064 \mathrm{mg}$ q8wk] with administration of the 1-day initiation regimen (a single dose of $\mathrm{AL}_{\mathrm{NCD}}$ and a single $30 \mathrm{mg}$ dose of oral aripiprazole) on the same day. In addition, simulations were carried out where AL (all approved doses) was administered 1, 3, 7, 10, or 14 days after the 1-day initiation regimen. AL treatment was continued at the prescribed dosing interval from the point of the first administration.

Simulations also were conducted to evaluate the use of $\mathrm{AL}_{\mathrm{NCD}}$ as an alternative to the current recommendation of daily oral aripiprazole supplementation to re-establish therapeutic aripiprazole plasma concentrations after a missed dose of AL. Re-establishment regimens $\left(\mathrm{AL}_{\mathrm{NCD}}\right.$ or 7-day oral aripiprazole) were begun simultaneously with the late AL dose. Simulations were performed to predict likely aripiprazole concentration-time profiles after multiple-dose scenarios to determine the likely impact of a late $\mathrm{AL}$ dose with and without supplementation with $\mathrm{AL}_{\mathrm{NCD}}$. Five $\mathrm{AL}$ dosing regimens were simulated (441, 662, and $882 \mathrm{mg} \mathrm{q4wk;} 882 \mathrm{mg} \mathrm{q6wk} ; 1064 \mathrm{mg} \mathrm{q} 8 \mathrm{wk}$ ) at steady state. A dose of AL was administered 1, 2, 3, 4, or 6 weeks late, either alone or supplemented with 7 days of oral aripiprazole or $\mathrm{AL}_{\mathrm{NCD}}$.

\section{Results}

\subsection{PopPK Model}

\subsubsection{Base Model and Covariate Analysis}

The base model was parameterized to describe aripiprazole after single IM administration of $\mathrm{AL}_{\mathrm{NCD}}$, single IM administration of AL, and oral aripiprazole administration from Studies 1, 2, and 3. The model contained central and peripheral compartments for aripiprazole and 16 structural parameters (further details are available in Supplementary Text, Online Resource 2). After $\mathrm{AL}_{\mathrm{NCD}}$ administration, conversion of IM $\mathrm{AL}_{\mathrm{NCD}}$ to aripiprazole was described by 
a double Weibull function. After AL administration, conversion of IM AL to aripiprazole was described by a zero-order process with the AL IM duration of absorption (Dur) estimated and the first-order absorption of aripiprazole from the dosing depot defined as 1/Dur. Additionally, a lag time (ALAG) from the IM depot to the appearance of aripiprazole in the central compartment was also present in the model.

The previous PopPK model for aripiprazole, after administration of $\mathrm{AL}$ and oral aripiprazole, included covariate terms to describe increased apparent volume of aripiprazole central compartment $(\mathrm{VC} / \mathrm{F})$ with body weight (power model fixed at allometric exponent of 1.0) and reduced apparent clearance of aripiprazole (CL/F) for CYP2D6 poor metabolizers [11]. For CYP2D6, the data set contained extensive metabolizers (EMs) and intermediate metabolizers (IMs) as well as patients whose phenotype was inconclusive; PMs were absent. An eta box plot for $\mathrm{CL} / \mathrm{F}$ versus CYP2D6 did not indicate any notable difference for non-EMs. Analyses conducted early in model development for Study 1 data alone showed an insignificant change in OFV when CL/F for non-EMs was evaluated. Consequently, the CYP2D6 phenotype was not evaluated for the current data set. Based on the eta versus covariate plots, weight was tested on VC/F (power model fixed at allometric exponent of 1.0), CL/F, AL Dur, and ALAG. Additionally, the potential effects of age on CL/F, $\mathrm{AL}_{\mathrm{NCD}}$ injection site on Weibull fraction of dose (FRAC), and AL injection site on AL Dur and ALAG were evaluated. For injection site effects, the change in parameter after administration in the deltoid was estimated relative to gluteal administration. Due to the limited number of Hispanic or Latino patients (5\%), ethnicity was not included in the covariate analysis. Race and sex were not included because there were no apparent differences in model parameter estimates based on these factors.

The base model was updated to include 8 covariate effects to form a full covariate model; estimates of these effects are presented in Supplementary Table S2 (Online Resource 1). Parameters associated with AL administration were consistent with those of earlier analyses [11]. Of the 8 effects in the model, 2 of the estimated injection site effects included the null value $\left(\mathrm{AL}_{\mathrm{NCD}}\right.$ injection site on FRAC and AL injection site on ALAG) and were removed. Removal of age on CL/F, weight on ALAG, weight on AL Dur, AL injection site on AL Dur, and weight on CL/F resulted in insignificant changes in the OFV. Thus, the model resulting from backward elimination contained just the single covariate effect of increasing $\mathrm{VC} / \mathrm{F}$ with weight.

\subsubsection{Final PopPK Model}

Data from Study 4 were added (to include data from AL administration alone) to develop the final PopPK model. A 2-compartment model with central and peripheral compartments for aripiprazole and conversion of IM AL to aripiprazole, described by a zero-order process with the duration of conversion estimated and the first-order absorption of aripiprazole from the dosing depot defined as 1/Dur, was used (Supplementary Fig. S1, Online Resource 3). Additionally, ALAG from the IM AL depot to the appearance of aripiprazole in the central compartment was included in the model. First-order processes described the absorption of aripiprazole after oral dosing and the movement between central and peripheral compartments in accordance with the previous model. However, the previous model was updated to include a double Weibull function that described the conversion of $\mathrm{IM} \mathrm{AL}_{\mathrm{NCD}}$ to aripiprazole in plasma following $\mathrm{IM} \mathrm{AL}_{\mathrm{NCD}}$ administration.

The final model parameters are presented in Supplementary Table S3 (Online Resource 1). Goodness-of-fit analyses demonstrated that the observed concentrations were well described by model predictions with no apparent study, administration route, or observed dose-effect biases (Supplementary Fig. S2; Online Resource 4). PcVPC plots were created by study for Study 1 and Study 3, and by regimen in Study 2 and Study 4. The pcVPC plot for the final model for Study 2, the only study to employ the intended initiation regimen, is shown in Supplementary Fig. S3 (Online Resource 5). The pcVPC plots indicated that the majority of observed concentrations were contained within the final PopPK model-predicted 90\% PIs within and across studies for Study 1 and Study 3, and by regimen within Study 2 and Study 4. The final PopPK model was deemed adequate to perform simulations evaluating various dosing and administration scenarios.

\subsection{Model Refinement for Simulations}

A mixture model was applied to patients receiving the 21-day initiation regimen in Study 2 to account for a subgroup of patients with lower exposure after multiple oral aripiprazole administrations over the first 28 days of dosing (Supplementary Text, Online Resource 2; Supplementary Fig. S4, Online Resource 6). The mixture model estimated a subpopulation of patients [37.4\%; $95 \%$ confidence interval (CI), 22.3-55.4\%] who had $44.6 \%$ lower oral bioavailability $\left(\mathrm{F}_{\mathrm{po}}\right)(95 \% \mathrm{CI} 40.9-48.3 \%)$ in the 21-day initiation regimen groups in Study 2. These estimates of reduction in $\mathrm{F}_{\mathrm{po}}$ and proportions of patients in the subpopulation from the mixture model were applied to the final PopPK model used for simulations. 
Fig. 1 Median simulated aripiprazole concentrations of simultaneous treatment of the 1-day initiation regimen with all approved AL 5 dosage regimens (left panel), and observed aripiprazole concentrations for all approved AL dosage regimens at Week 12 (data from previous studies; right panel) [6, 11-13]. The boxes represent the 25 th and 75th percentiles of aripiprazole concentration, the line within each box marks the median, the asterisk indicates the mean, and the whiskers indicate the 10th and 90th percentiles. The circles represent individual observations beyond the 90 th percentiles. $A L$ aripiprazole lauroxil, $A L_{N C D}$ aripiprazole lauroxil nanocrystal dispersion, $q X w k$ every 4,6 or 8 weeks

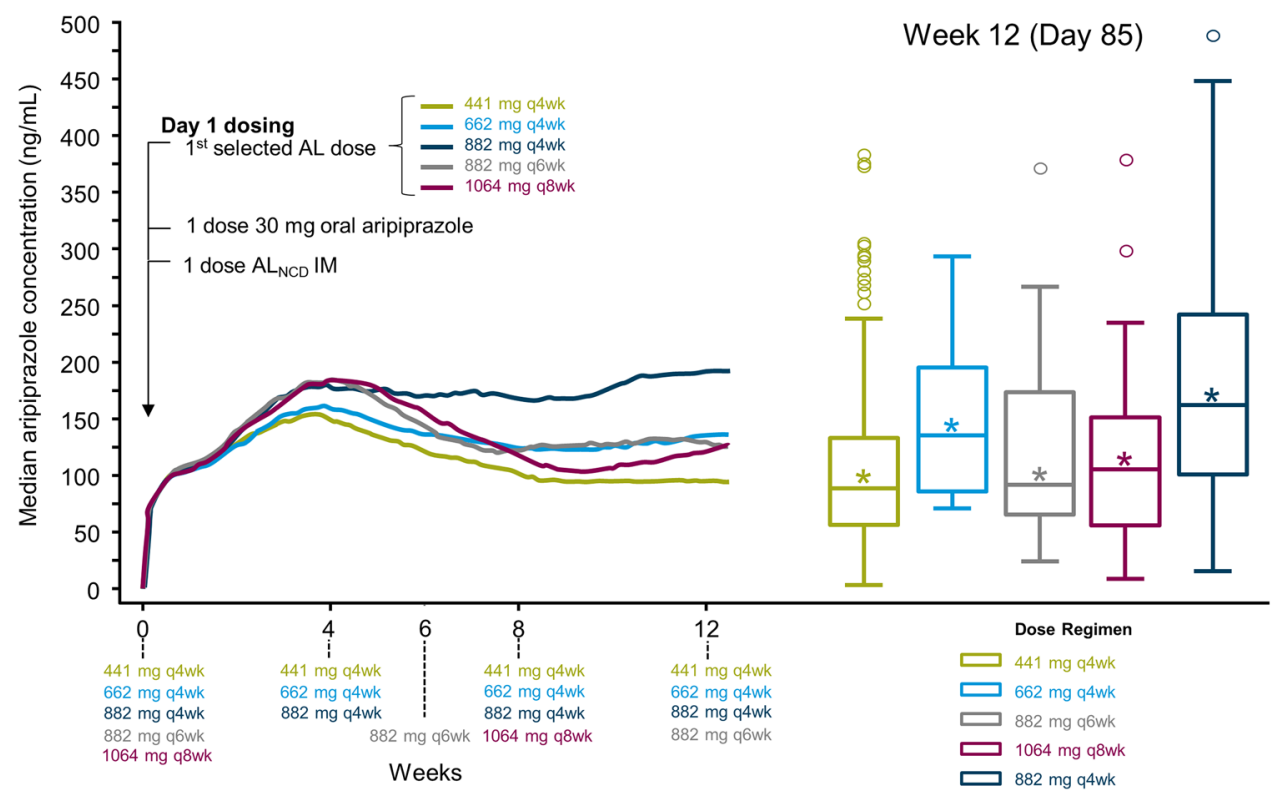

\subsection{Model Simulations}

\subsubsection{Simultaneous Initiation with AL and 1-Day Initiation Regimen}

The observed results of Study 2 demonstrated that coadministration of the 1-day initiation regimen with AL 441 or $882 \mathrm{mg}$ achieved aripiprazole concentrations within the range associated with therapeutic doses of aripiprazole within 4 days, similar to those of the 21-day initiation regimen administered in conjunction with the first AL dose [9]. Simulations were conducted for all approved AL dosing regimens (441, 662, $882 \mathrm{mg} \mathrm{q4wk;} 882 \mathrm{mg} \mathrm{q6wk;} 1064 \mathrm{mg}$ q8wk) with the 1-day initiation regimen given the same day as the starting dose of AL. Median simulated aripiprazole concentrations over time for the first 12 weeks are shown in Fig. 1. In all cases, same day administration of AL with the 1-day initiation regimen was predicted to achieve aripiprazole concentrations within the range associated with therapeutic doses of $\mathrm{AL}$ using the 21-day initiation regimen (Fig. 1, right panel) within 4 days of treatment initiation, which were sustained throughout the initiation period. Aripiprazole concentrations remained comparable for all 5 $\mathrm{AL}$ dosing regimens in the first 2 weeks. After the first 2 weeks, AL dose-related differences in aripiprazole concentrations started to become apparent, as expected, and were consistent with those observed in earlier studies [11].

\subsubsection{Administration of First AL Dose After the 1-Day Initiation Regimen}

Simulations were conducted to evaluate whether the 1-day initiation regimen could be administered on a separate day earlier than the first dose of AL. All 5 AL dosing regimens were again simulated $(441,662,882 \mathrm{mg}$ q4wk; $882 \mathrm{mg}$ q6wk; $1064 \mathrm{mg} \mathrm{q8wk).} \mathrm{For} \mathrm{all} \mathrm{scenarios} \mathrm{the} \mathrm{1-day} \mathrm{initiation}$ regimen was administered on day 0 , and the first $\mathrm{AL}$ dose was given on the same day as the 1-day initiation regimen or $1,3,7,10$, or 14 days later (i.e., starting AL on days 1 , $3,7,10$, and 14). AL was then continued at the prescribed dosing interval from that point forward.

Delaying the start of $\mathrm{AL}$ administration relative to the 1-day initiation regimen reduced median aripiprazole concentrations over the first 2 dosing intervals of $\mathrm{AL}$ compared with simultaneous treatment initiation, and the magnitude of this effect was determined by the length of the delay. When AL was administered 1 or 3 days after the 1-day initiation regimen, negligible decreases in aripiprazole concentrations were observed at the end of the $\mathrm{AL}$ dosing interval $(\mathrm{C} \tau)$ compared with when AL was administered on the same day as the 1-day initiation regimen. Delays in the first $\mathrm{AL}$ injection $\geq 1$ week from the 1-day initiation regimen were also assessed. When AL was administered 7, 10, or 14 days later, the median aripiprazole $\mathrm{C} \tau$ (across all AL regimens) was lower by 8-16, 9-23, and 14-31\%, respectively, compared with same day administration. Simulated median aripiprazole concentrations for staggered treatment initiation of the 1-day initiation regimen and AL administered 10 days later are shown in Fig. 2 for all doses. Administration of AL 10 days after the 1-day initiation regimen predicted aripiprazole $\mathrm{C} \tau \geq 77 \%$ of that attained with same day administration. This was still considered to be within the concentration range associated with therapeutic doses of AL. 
Fig. 2 Median simulated aripiprazole concentrations for administration of $\mathrm{AL}$ on the same day as or 10 days after the 1-day initiation regimen. a AL $441 \mathrm{mg}$ q4wk b AL $662 \mathrm{mg}$ q4wk c AL 882 mg q4wk d AL 882 mg q6wk e AL 1064 mg q8wk. The 1-day initiation regimen $\left(\mathrm{AL}_{\mathrm{NCD}}\right.$ and $30 \mathrm{mg}$ oral aripiprazole) was administered on day 0 in all simulations. $A L$ aripiprazole lauroxil, $A L_{N C D}$ aripiprazole lauroxil nanocrystal dispersion, $q X w k$ every 4,6 or 8 weeks (a)

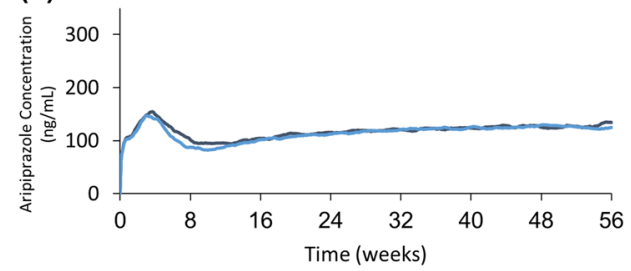

(c)

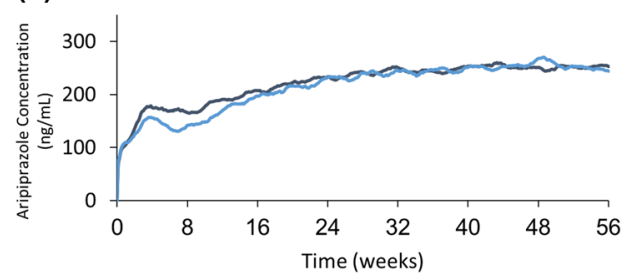

(e)

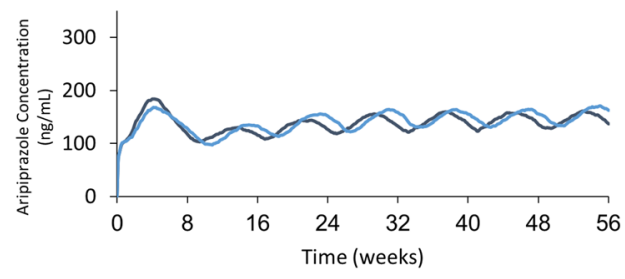

(b)

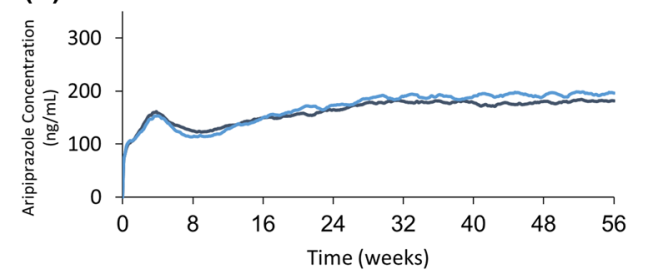

(d)

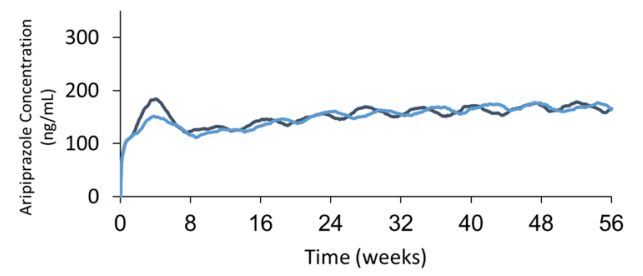

First AL injection on Day 0 (1-day) First AL injection on Day 10 (10-day delay)

\subsubsection{Re-establishment of Therapeutic Aripiprazole Concentrations Following a Missed Dose of AL}

Simulations were performed to evaluate the use of $A L_{N C D}$ as an alternative to the recommended 7-day oral aripiprazole supplementation to re-establish aripiprazole concentrations within the range associated with therapeutic doses of $\mathrm{AL}$ after a missed dose of $\mathrm{AL}$ [4]. All approved $\mathrm{AL}$ dosing regimens were simulated (441, 662, $882 \mathrm{mg}$ q4wk; $882 \mathrm{mg} \mathrm{q} 6 \mathrm{wk} ; 1064 \mathrm{mg} \mathrm{q} 8 \mathrm{wk})$ at steady state. A dose of AL was administered 1, 2, 3, 4, or 6 weeks late (depending on the dosing regimen) alone, with 7 days of supplemental oral aripiprazole, or with a single injection $\mathrm{AL}_{\mathrm{NCD}}$ (without the single oral aripiprazole dose).

Median simulated aripiprazole concentrations for selected late dosing scenarios for the 5 approved $\mathrm{AL}$ regimens (with and without the re-establishment regimens) are presented in Table 2. These scenarios require 7 days of oral aripiprazole supplementation with the late $\mathrm{AL}$ dose to restore aripiprazole concentrations within the range associated with therapeutic doses of AL [4]. In these simulations, as expected, late administration of the AL dose resulted in lower median predicted concentrations than when administration of the $\mathrm{AL}$ dose was not delayed. When $\mathrm{AL}_{\mathrm{NCD}}$ was used in conjunction with $\mathrm{AL}$ after a missed dose, aripiprazole concentrations increased and reached maximal drug concentrations $\left(\mathrm{C}_{\max }\right)$ similar to those achieved with 7 days of oral aripiprazole and spanned the maximal concentration range associated with the approved dose range of $\mathrm{AL}$.
When AL doses were taken as scheduled, aripiprazole $\mathrm{C}_{\max }$ ranged from 153 to $310 \mathrm{ng} / \mathrm{mL}$. Both re-establishment regimens resulted in $\mathrm{C}_{\max }$ values within a range comparable to that of $\mathrm{C}_{\max }$ values for $\mathrm{AL}$ at steady state and, thus, were adequate at restoring aripiprazole concentrations within the range associated with therapeutic doses of AL. These simulations demonstrated that when an AL dose is missed, coadministration with either 7 days of oral aripiprazole or a single dose of $\mathrm{AL}_{\mathrm{NCD}}$ is effective in returning aripiprazole concentrations within the range associated with therapeutic doses of AL.

Therefore, $\mathrm{AL}_{\mathrm{NCD}}$ is a suitable alternative to 7 days of oral aripiprazole supplementation for recovery of aripiprazole concentrations after a missed AL dose. Further simulations (data not shown) indicated that longer delays (requiring 21 days of oral aripiprazole supplementation according to the current prescribing information [4]) would require resumption of $\mathrm{AL}$ treatment with the 1-day initiation regimen.

\section{Discussion}

Based on an existing model, a PopPK model of aripiprazole has been developed that accurately describes concentration-time profiles of aripiprazole after IM administration of $\mathrm{AL}_{\mathrm{NCD}}$ and $\mathrm{AL}$ and after oral administration of aripiprazole in patients with schizophrenia. There appeared to be a distinct grouping of patients in the $15 \mathrm{mg}$ oral aripiprazole (21-day initiation regimen) arms of Study 2 who had lower 
Table 2 Summary of simulated aripiprazole $\mathrm{C}_{\max }$ values resulting from a late dose with 7-day oral aripiprazole or $\mathrm{AL}_{\mathrm{NCD}}$ recovery and with no late dose

\begin{tabular}{|c|c|c|c|c|c|}
\hline \multirow[t]{3}{*}{ Dose regimen } & \multirow[t]{3}{*}{ Time since last dose, weeks } & \multirow[t]{3}{*}{ Statistic } & \multicolumn{3}{|l|}{$\mathrm{C}_{\max }(\mathrm{ng} / \mathrm{mL})$} \\
\hline & & & \multirow[t]{2}{*}{ No late dose } & \multicolumn{2}{|l|}{ Late dose and recovery } \\
\hline & & & & 7-Day $15 \mathrm{mg}$ oral aripiprazole & $\mathrm{AL}_{\mathrm{NCD}}$ \\
\hline \multirow[t]{3}{*}{$441 \mathrm{mg} \mathrm{q} 4 \mathrm{wk}$} & \multirow[t]{3}{*}{6} & Median & 153.0 & 156.4 & 224.2 \\
\hline & & 25th percentile & 113.6 & 117.5 & 163.7 \\
\hline & & 75th percentile & 203.4 & 218.6 & 306.4 \\
\hline \multirow[t]{3}{*}{$662 \mathrm{mg} \mathrm{q} 4 \mathrm{wk}$} & \multirow[t]{3}{*}{8} & Median & 227.1 & 218.8 & 266.9 \\
\hline & & 25th percentile & 165.0 & 167.7 & 181.2 \\
\hline & & 75th percentile & 311.2 & 296.8 & 361.8 \\
\hline \multirow[t]{3}{*}{$882 \mathrm{mg} \mathrm{q} 4 \mathrm{wk}$} & \multirow[t]{3}{*}{8} & Median & 309.6 & 286.5 & 325.8 \\
\hline & & 25th percentile & 226.9 & 202.1 & 234.7 \\
\hline & & 75th percentile & 446.9 & 394.4 & 439.5 \\
\hline \multirow[t]{3}{*}{$882 \mathrm{mg} \mathrm{q6wk}$} & \multirow[t]{3}{*}{8} & Median & 226.2 & 226.4 & 251.2 \\
\hline & & 25th percentile & 167.1 & 163.5 & 180.5 \\
\hline & & 75th percentile & 333.1 & 309.8 & 351.4 \\
\hline \multirow[t]{3}{*}{1064 mg q8wk } & \multirow[t]{3}{*}{10} & Median & 209.5 & 211.6 & 217.4 \\
\hline & & 25th percentile & 148.2 & 155.3 & 158.2 \\
\hline & & 75th percentile & 292.3 & 297.5 & 305.3 \\
\hline
\end{tabular}

$q 4 w k$ every 4 weeks, $q 6 w k$ every 6 weeks, $q 8 w k$ every 8 weeks, $C_{\max }$ maximum drug concentration

exposure following multiple administrations of oral aripiprazole. Therefore, a mixture model was applied to only their data to formally evaluate whether a subpopulation with lower $\mathrm{F}_{\mathrm{po}}$ was evident. Applying the estimated reduction in $\mathrm{F}_{\mathrm{po}}$ and the proportion of patients in the subpopulation resulted in aripiprazole concentrations after multiple oral administrations that were more consistent with those observed in this and other studies.

Overall, the final model parameter estimates and covariates for $\mathrm{AL}$ and oral aripiprazole were consistent with those from the previous PopPK models $[11,12]$ and were deemed adequate to perform simulations evaluating various dosing and administration scenarios.

Earlier clinical trial results and model-based simulations in the present study provide evidence that the 1-day initiation regimen in combination with the first $\mathrm{AL}$ injection (at all approved dosing regimens) results in aripiprazole concentrations within the range associated with therapeutic doses of AL within 4 days, and exposures in the first month of AL treatment initiation comparable to those achieved with the 21-day initiation regimen. The model-based simulations also demonstrate flexibility in the use of the 1-day initiation regimen, in that the first $\mathrm{AL}$ injection can be administered up to 10 days later than the 1-day initiation regimen, while retaining predicted plasma aripiprazole exposures within the range associated with therapeutic doses of AL.

A previous study had demonstrated that the prolonged exposure to aripiprazole provided by $\mathrm{AL}$ offers an additional window of time before oral aripiprazole supplementation is required on resumption of $\mathrm{AL}$ dosing following a delay [12]. However, in the event of longer dose delays, 7 days of oral aripiprazole supplementation are required with the late $\mathrm{AL}$ dose to restore aripiprazole concentrations within the range associated with therapeutic doses of AL [4]. The current model-based simulations suggest that $A L_{N C D}$ is a suitable alternative to 7 days of oral aripiprazole supplementation for re-establishment of aripiprazole concentrations after a missed $\mathrm{AL}$ dose. The flexibility of use of $\mathrm{AL}_{\mathrm{NCD}}$ may be particularly helpful for patients who have problems with treatment adherence.

A limitation of this analysis is that the findings would not apply to CYP2D6 PMs. It is noted that most of the patients were African American; however, the lack of apparent differences in model parameter estimates based on race suggests that findings of this study are generalizable across different populations. Another limitation is that the conclusions regarding delayed AL initiation and missed dosing are based on simulated data. Although the model performance was validated, the clinical impact based on recommendations coming from simulated results has not been verified in clinical studies. Nonetheless, modeling and simulation are important tools for drug development because they provide the opportunity to evaluate dosing strategies in scenarios that frequently occur in clinical practice but would otherwise not be evaluated in clinical trials. 


\section{Conclusions}

Pharmacokinetic data in this report provide evidence and a rationale for the use of $\mathrm{AL}_{\mathrm{NCD}}$ for the initiation of $\mathrm{AL}$ treatment and the management of missed AL doses. The flexibility of the 1-day initiation regimen provides opportunities to tailor the start of AL therapy to best meet the needs of the individual patient.

Acknowledgments The authors thank all patients and investigators who participated in the clinical trials that were included in the population pharmacokinetics analysis. Medical writing and editorial support for the preparation of this manuscript (under the guidance of the authors) was provided by Karen Yee (ApotheCom, UK).

\section{Compliance with Ethical Standards}

Funding This study was sponsored by Alkermes, Inc., Waltham, MA, USA. Funding for editorial support for manuscript preparation was provided by Alkermes, Inc., Waltham, MA, USA.

Conflict of interest Marjie Hard is a former employee of Alkermes, Inc. Angela Wehr and Lisa von Moltke are employees of Alkermes, Inc. Brian Sadler and Richard Mills are employees of ICON plc who performed the work under contract to Alkermes, Inc.

Open Access This article is distributed under the terms of the Creative Commons Attribution-NonCommercial 4.0 International License (http://creativecommons.org/licenses/by-nc/4.0/), which permits any noncommercial use, distribution, and reproduction in any medium, provided you give appropriate credit to the original author(s) and the source, provide a link to the Creative Commons license, and indicate if changes were made.

\section{References}

1. Weiden PJ, Kozma C, Grogg A, Locklear J. Partial compliance and risk of rehospitalization among California Medicaid patients with schizophrenia. Psychiatr Serv. 2004;55:886-91.
2. Heslin KC WA. Hospital Readmissions Involving Psychiatric Disorders, 2012. HCUP Statistical Brief \#189. 2015. http://www. hcup-us.ahrq.gov/reports/statbriefs/sb189-Hospital-ReadmissionsPsychiatric-Disorders-2012.pdf. Accessed 25 May 2018.

3. Brissos S, Veguilla MR, Taylor D, Balanzá-Martinez V. The role of long-acting injectable antipsychotics in schizophrenia: a critical appraisal. Ther Adv Psychopharmacol. 2014;4:198-219.

4. Alkermes Inc. Aristada (Aripiprazole Lauroxil) Prescribing Information. 2018. https://www.aristadahcp.com/downloadables/ ARISTADA-PI.pdf. Accessed 25 May 2018.

5. Cruz MP. Aripiprazole lauroxil (Aristada): an extended-release, long-acting injection for the treatment of schizophrenia. Pharm Ther. 2016;41:556-9.

6. Meltzer HY, Risinger R, Nasrallah HA, Du Y, Zummo J, Corey $\mathrm{L}$, et al. A randomized, double-blind, placebo-controlled trial of aripiprazole lauroxil in acute exacerbation of schizophrenia. J Clin Psychiatry. 2015;76:1085-90.

7. Raoufinia A, Baker RA, Eramo A, Nylander AG, Landsberg W, Kostic D, et al. Initiation of aripiprazole once-monthly in patients with schizophrenia. Curr Med Res Opin. 2015;31:583-92.

8. Raoufinia A, Peters-Strickland T, Nylander AG, Baker RA, Eramo A, Jin N, et al. Aripiprazole once-Monthly $400 \mathrm{mg}$ : comparison of pharmacokinetics, tolerability, and safety of deltoid versus gluteal administration. Int J Neuropsychopharmacol. 2017;20:295-304.

9. Hard ML, Wehr AY, Du Y, Weiden PJ, Walling D, Von Moltke L. Pharmacokinetic evaluation of a one-day treatment initiation option for starting long-acting aripiprazole lauroxil for schizophrenia. J Clin Psychopharmacol. 2018; manuscript in press.

10. Ahn JE, Karlsson MO, Dunne A, Ludden TM. Likelihood based approaches to handling data below the quantification limit using NONMEM VI. J Pharmacokinet Pharmacodyn. 2008;35:401-21.

11. Hard ML, Mills RJ, Sadler BM, Wehr AY, Weiden PJ, von Moltke L. Pharmacokinetic profile of a 2-month dose regimen of aripiprazole lauroxil: a phase I study and a population pharmacokinetic model. CNS Drugs. 2017;31:617-24.

12. Hard ML, Mills RJ, Sadler BM, Turncliff RZ, Citrome L. Aripiprazole lauroxil: pharmacokinetic profile of this long-acting injectable antipsychotic in persons with schizophrenia. J Clin Psychopharmacol. 2017;37:289-95.

13. Alkermes Inc. ALK9072-002 (Unpublished data). 